Article

\title{
Demand Response and Distributed Generation Remuneration Approach Considering Planning and Operation Stages
}

\author{
Cátia Silva ${ }^{1}\left(\mathbb{D}\right.$, Pedro Faria $^{1, *(1)}$ and Zita Vale ${ }^{2}$ (1) \\ 1 GECAD—Research Group on Intelligent Engineering and Computing for Advanced Innovation and \\ Development, Rue Dr.Antonio Bernardino de Almeida 431, 4200-072 Porto, Portugal \\ 2 Polytechnic of Porto, Praça de Gomes Teixeira, 4099-002 Porto, Portugal \\ * Correspondence: pnf@isep.ipp.pt; Tel.: +351-228-340-511; Fax: +351-228-321-159
}

Received: 22 April 2019; Accepted: 10 July 2019; Published: 16 July 2019

check for updates

\begin{abstract}
The need for new business models to replace existing ones, soon obsolete, is a subject often discussed among researchers in the area. It is essential to find a practical solution that includes the concepts of demand response and distributed generation in the energy markets, these being the future of the electricity grid. It is believed that these resources can bring advantages to the operation of the system, namely increasing technical efficiency. However, one of the problems is the aggregation of small resources as a result of the associated uncertainties. The authors propose a business model with three main phases used in planning: optimal scheduling, aggregation, and remuneration. In this paper, a new phase was added, the classification, with the main purpose of assisting the aggregator of these small resources in operating situations. The focus is on the fair remuneration of participants in the management of the market, in addition to minimizing operating costs. After testing four different remuneration methods, it was proved that the method proposed by the authors obtained better results, proving the viability of the proposed model.
\end{abstract}

Keywords: classification; demand response; remuneration; energy market

\section{Introduction}

Currently, the demand response (DR) concept is inserted in almost all the studies related with business models from the current electricity market and even with the current electricity grid. This concept is something that can be considered very interesting for both consumers and system operators as it could be crucial in peak load conditions. It is considered that in order to exploit DR's full potential, will be necessary to also integrate system concepts the distributed generation (DG), namely renewable energy resources (RES) such as wind and solar, and a more effective, reliable, and cheap communication with improvements in the infrastructures and used technologies [1]. However, this integration in the actual business models is still scarce and no European country has yet managed to apply these concepts entirely in their market [2]. In this way, it is essential to find solutions that incorporate these resources, enabling the communication between them, to be possible for the system to flow in a safe, sustainable, and effective way.

DR programs for small-scale resources are also poorly explored in energy markets and business models, despite efforts to highlight their importance. These features may be useful when their response is coordinated and when associated with an aggregator-virtual power player (VPP) [3]. VPP becomes responsible for the coordinated management of the small resources associated with and will participate in wholesale electricity markets as an intermediary in the transactions with the independent system operator. The authors believe that following this approach, the system 
becomes more flexible-increasing the communication in order to achieve bi-directionality, influence on operating costs, and increased reliability by allowing greater penetration of small-scale renewable resources. However, for this approach to work fully, the role of the aggregator cannot be neglected in the system, as it is very important for the optimal management of these resources. The authors suggest an optimal scheduling of all the resources, through one of the phases of the proposed methodology. The proposed methodology can produce viable results both with a small database of consumers, DG units, and even prosumers, as well as a database composed with thousands of these resources. The analysis presented in the present paper used an approach that includes all the mentioned resources, but will focus on the consumers participating in incentive-based DR (IDR) programs, studying in detail all the phases that compose the methodology. It should be noted, however, that aggregators of one type of resource may also make use of this solution.

Knowing that the uncertainty of participation in the market transactions of these small resources is high, the remuneration and the incentive for the continuous participation becomes a very relevant subject. The aggregator, having direct communication, should find appropriate methods and tools to remunerate the associated resources fairly. The authors of the present paper consider that the formation of groups of resources with similar characteristics becomes an option. Several studies point to clustering methods as viable algorithms to perform this type of aggregation. Through a set of information, these methods can identify patterns between objects and thereby form groups. The authors subdivide clustering methods into partitioning, fuzzy clustering, hierarchical clustering, and density-based or model-based methods [4]. In the proposed methodology, the main goal of this aggregation will be the formation of groups so that in the next phase, remuneration, it would be possible to find the better group's tariff in a way that all resources receive according to their contribution (for consumers reduction of the load and for the producers the availability). As all elements have similar characteristics, the final tariff will be fair. There will be the possibility, for example, of benefiting small resources. As the methodology can integrate different types and sizes of consumers, if small- and medium-sized groups are formed-remembering that they will always depend on their contribution-the tariff will be higher for the small resource having a higher incentive.

By introducing a dynamic remuneration tariff, the authors propose a different formation from previous works: the remuneration tariff should be formed by the sum of a fixed rate, derived from the contract with the VPP; and a rate that depends on the market (indexed tariff). It is recalled, however, that the remuneration tariff is affected by the seasonality. The study for different time frames will be important, encouraging the discussion and comparison of several time horizons to find the solution that best suits the situation where the VPP is.

The methodologies presented so far may serve as a planning case with all the aggregated resources foreseeing its consumption, or generation. In the case of real-time operation, the possibility of introducing a resource in a remuneration group without going through all the required task needs can be useful. In this way, the authors aimed to find a solution that contemplates the same phases, but in a short time and with a lower computation burden. The authors propose the application of classification methods for this type of situation. Classification is considered the task of assigning an object from a database to a class or group, taking into account a set of characteristics that describe the mention object. Decision trees (DT) are considered a method with a lot of potential in the classification area [5]. As the name suggests, this method uses a tree structure to class objects by dividing the dataset into smaller subsets. For the present paper and using previous results to create many decision trees, it will be possible for the VPP to introduce, at that moment (real-time operation), considering only the results from the optimization, each resource in the respective remuneration group, being compensated with the respective remuneration tariff. 
When compared with previous works, several upgrades towards contribution and innovation aspects were done in this paper:

- Real-time approach is applied in the present paper with the classification method. In the work of [3], the authors focus in a planning perspective with the approach proposed. In this paper, the authors went further and designed a decision tree where the VPP can apply the methodology to briefer time horizon without going through all the methodology phases. With this innovation, the VPP can attribute to a player a remuneration group and the respective tariff in a real-time tactic and with the scenarios created for the effect;

- A different time horizon was considered. In the work of [3] 2 to 8 January 2018 and in this paper between 1 and 7 February 2019 presenting dissimilar database value resulting in different solutions;

- Regarding the remuneration methods, the authors of [3] study the impact of dynamic tariffs, considering a parameter with the market price for the mention day varying the percentage of influence in the final tariff. In the present paper, taking into account this previous study, the authors tested the only the percentage that the VPP has more benefit, but still maintaining the fair remuneration for the participation;

- In fact, another upgrade when compared with the work of [3] was attributing a minimum value for the DG units be used. Some technologies may not be worth having available in situation with a lower amount of production. In this way, with the new equation introduced in the optimization phase, the DG units are bounded with a lower limit;

- Although the comparison between the two methods to find the optimum $\mathrm{k}$ for the clustering method in the aggregation phase was already performed in the work of [3], the authors consider important additional tests to a deeper understanding.

- In the present paper, a higher emphasis was given to the scheduling phase regarding the work of [3]. Given that the real-time operation proposed only performed two phases, scheduling and classification, the results of the optimization were more detailed.

The organization of this paper is divided into six sections. Section 1 is an introduction to the topic addressed. Section 2 presents a review of the current literature. Section 3 presents the methodology proposed by the authors in a more detailed way. Section 4 presents the case study chosen to prove the feasibility of the methodology and the results are presented in Section 5. Finally, Section 6 presents the conclusions drawn from the study.

\section{Related Literature}

In the related literature, one can find several approaches to this problem. Focusing on optimization, in the work of [6], a cost minimization problem has been formulated for microgrids that are equipped with unstable generation resources, namely renewable energy sources and combined heat and power (CHP) generators. In the work of [7], an optimization scheduling framework for systems with high penetration of wind power was proposed, incorporating demand response programs, as well as in the work of [8], but focusing on the influence of wind power uncertainty. In another paper [9], there is an initial version of the proposed methodology which was developed for a single period only.

Proceeding the remuneration, in other research [10], consumers are remunerated individually through dynamic pricing, while in the work of [11], a model is presented for a retailer that considers DR and the uncertainty of the market price by combining the Stackerlber game with a linear problem in the definition of the price. The benefit of DR participants is maximized in another paper [12] through the daily consumption information and preferences. In the work of [13], an incentive mechanism for the demand side management using auction is presented.

Several technics have been used to support the remuneration and its negotiation. In the works of $[14,15]$, methods are shown for energy scheduling based on cooperative game theory. The authors of [14] created a model that generation companies and different marketers can cooperate in order to maximize their profits. They inference that, with bilateral contracts, these agents can establish 
collaboration agreements that would be more profitable, despite the existence of natural competition. In the work of [15], the proposed model shows a simultaneous cost-optimal planning for controllable appliances with shared storage system and RES. This study explores the potential of an energy sharing system in order to reduce the individual user consumption cost while limiting the peak average ratio. Such approaches based on game theory and other negotiation methods are very relevant for the definition of an accurately fair remuneration of consumers participating in DR programs and events, in different contexts. In the present paper, the authors focus on proposing an approach that provides a remuneration tariff in which the consumer is not asked to make bids or to negotiate so those aspects can be a way that consumers will disregard DR programs preferring to keep their comfort.

Integrating storage units, in the literature [16-18], demand side management techniques are shown with consumers, producers, prosumers, as well as users with storage capability. In the work of [16], the model proposed can work with controllable appliances, RES, dispatchable energy resources, and energy storage systems. These authors report that with the increasing of the home smartness, the reduction of the energy cost is proven. The works of [17] and [18] take into account the uncertainties. Namely, in the work of [17], the model introduced manages energy storage systems and distributed generation, working with wind and solar generation uncertainties. In another paper [18], a predictive control model for real-time is presented, considering deferrable and non-deferrable load, RES, and energy storage systems with dynamic profiles. These studies were able to reduce the total user load in a sustainable way.

In the literature [19-21] models are introduced in which the harvested energy at one renewable energy generator or the stored energy at one storage system can be utilized to operate more than one user. To distinct goals exist in another paper [19]: quantify the optimal utilization of RES and evaluating how the volatility of RES influences the optimal exploitation. The authors proved that the users can benefit with deeper penetration of these technologies. In the work of [20], a group of interconnect users will buy their energy from producer and shared RES. The goal is to ensure social welfare and find the cost optimal planning of users with controllable appliances. Regarding another paper [21], Pilling et al. developed a model with joint operation of a microgrid and its hosting utility. The case study from the work of [21] proved that systems cost operation can be reduced by minimizing daily operation costs through energy exchange.

Considering the mentioned works, it can be mentioned that the proposed methodology presents some innovations. Regarding resources, the proposed methodology is able, regardless of the size of the database, to aggregate both small- and large-sized resources, while still counting on the prosumers. Still on the same basis, the VPP can aggregate different types of consumers, DG units, or the mixture of the two, making the method quite flexible. The fact of operating both in single period and in multiperiod optimization brings a huge benefit to VPP. Putting aside the competition in DR participation, the proposal is to form a fair tariff through groups rather than remunerate them individually. Summarizing, the main objective is the minimization of operating costs by VPP, adequately remunerating the resources that participate in the management of the market.

\section{Proposed Methodology}

Through the proposed methodology, the authors intend to investigate questions that could be essential in the definition of an effective business model and adapt to the future changes of the energy market. So, how does the formation of the tariff influence the remuneration of resources? Should this tariff be the same for every day of the week or change according to the time frame? What is the method that should be used for a real time approach? The methodology proposed by the authors seeks to answer all the questions presented, but the focus of the present paper will be to answer the last question. In previous works, the authors performed the methodology on a planning perspective. In this paper, the goal will be finding a way to apply the same method in real time. Thus, the authors take an approach that will include methods of classification. Through decision trees, the VPP will have the opportunity to choose the remuneration group for each resource associated considering the results 
from the optimization. In fact, because the methodology proposed by the authors gives the freedom to the VPP for choosing the interval of time, for the formation of this decision tree, a wider database can be used.

According to Figure 1, the proposed methodology presents different phases that support the decisions of the aggregator in the management of the market. This methodology also focuses on the fair remuneration of all the resources that participate in DR events wherever requested. This assumption is because the aggregator is an independent entity and that all associated resources obligatorily have a contract with this entity. Pointing to the different innovations that this proposal presents regarding the contributions of previous work already discussed in Section 2, emphasis is given to the following:

- Focus on small resources, underlining the introduction of prosumers as the junction of consumer and producer, presenting unique characteristics. The authors believe that their influence may be significant reduction of the load on DR programs;

- Creation of DR groups according to their actual response. The idea is to assemble resources with similar responses in the same group so that in the creation of remuneration groups, the tariff assumes a fair value. In this way, there may be groups composed of small- or medium-sized resources;

- The optimization and aggregation phases are performed for a given time frame, consecutively. Thus, the remuneration considers the performance of each of the resources or consumers for a whole set of days, being the distinction between working days and the weekend;

- Regarding the formation of the tariff, it is a mix between a fixed tariff and an indexed tariff. With this, the resources know that they always can count with the fixed part of the remuneration, being the value agreed in the contract. The indexed tariff considers the actual market prices. In this way, the VPP will have an increased risk, but it will benefit from the changes in the tariffs practiced in the market;

- There is also a discussion about the seasonality of tariffs throughout the week and even during the day for consumers. This fact adds a factor of variability to the methodology, thus increasing its interest;

- It should be noted that the proposed methodology, and all phases associated with it, can easily be used for a small database, as well as one that reaches thousands of resources. Remember, however, that there may be hardware limitation. Regarding the time to process the information, this approach is for the planning case and is driven to be run in a day-ahead or hour-ahead configuration;

- For real-time operation, the authors take another path, using classification methods, introducing the possibility of joining new resources or consumers according to their characteristics. In other words, considering the results from the optimization scheduling, the VPP can choose the right remuneration group for the resources without going through all phases again. This approach is the main innovation of this paper compared with other works;

- The comparison between the optimal and ideal number of clusters is discussed in such a way that VPP can determine the best number of DR programs to be implemented.

With this, the methodology proposed and presented in Figure 1 is composed of four main phases: scenario definition and information processing, optimal scheduling, aggregation, and remuneration. For this paper, as innovation from previous works, another phase was introduced to the methodology, classification, giving the final remuneration in a real time, without going through all the phases.

Starting with detailing the optimal scheduling phase, the objective function is to minimize operation costs. Consumers from DR programs, DG units, and external suppliers are considered, with two existing types of the last one: regular and additional. The suppliers will be used for the case of DG units not being able to meet the demand needs. Regarding demand, there are consumers who are associated with DR programs. These may belong to IDR programs being remunerated according to their contribution to community management, that is, in reducing the total load. For this phase, it will then be necessary to collect the input data, namely all the characteristics associated with the resources belonging to the aggregator. For the prosumers' case, it was considered that the final 
balance between generation and consumption is the considered value for the optimization, being remunerated accordingly.

The application of storage systems to this methodology could also be considered because these systems are very advantageous for the system. It would be considered that these resources would have an individual contract with the aggregator, such as DG units, even if associated with a consumer. In this way, all production is used to feed all the demand from the aggregate system of the VPP.

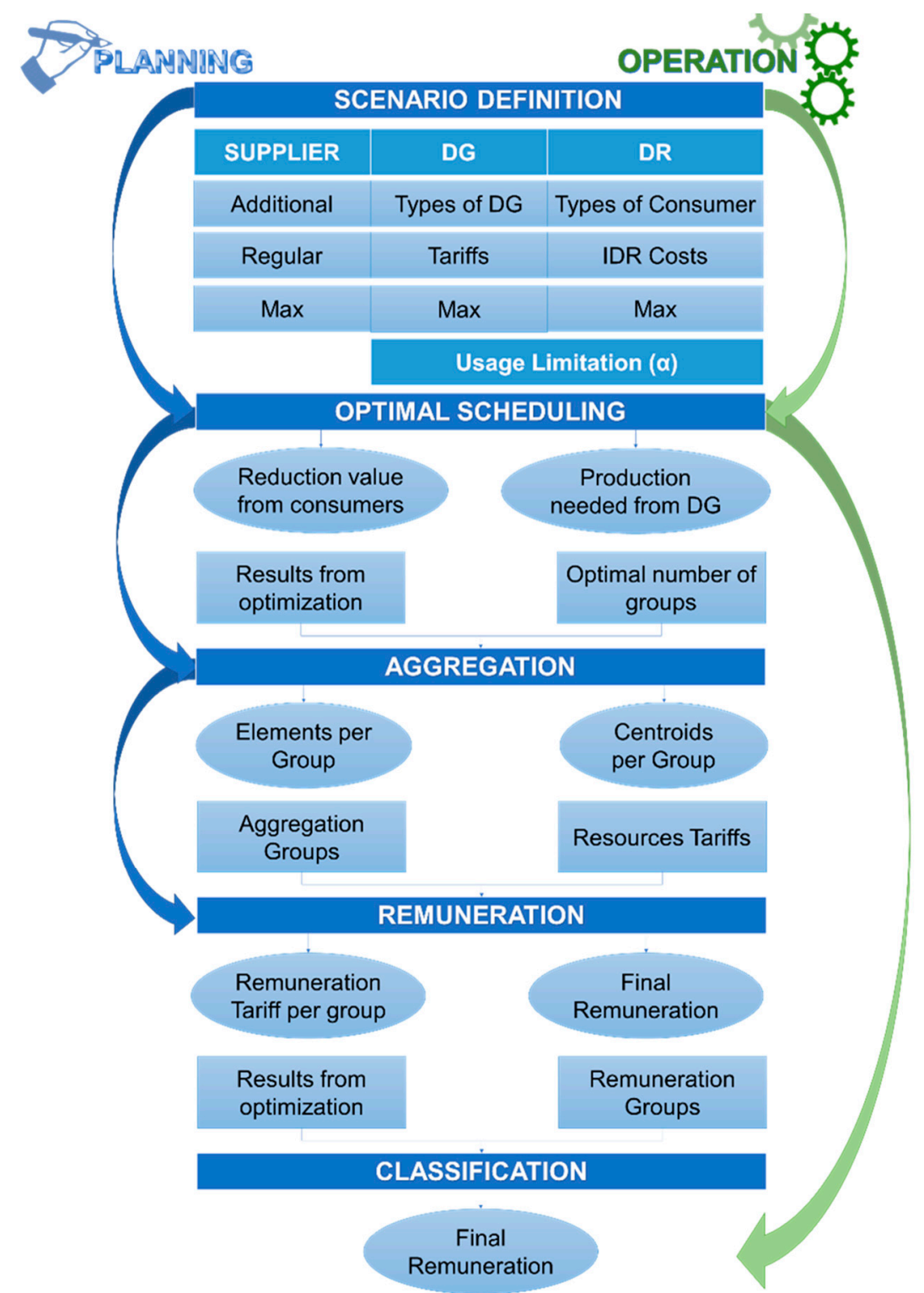

Figure 1. Diagram of the proposed methodology. DR, demand response; DG, distributed generation; IDR, incentive-based DR.

In the optimization performed, considering information like the maximum capacity of the DG units, the suppliers, and the reduction capacity of the consumers belonging to DR programs, as well as the consumption tariffs associated with each resource, is needed. Equation (1) presents the objective function of the problem. 


$$
\begin{array}{r}
\text { MinOC }=\sum_{p=1}^{P} P_{D G(p)} C_{D G(p)}+P_{N S P} C_{N S P}+\sum_{\text {sr }=1}^{S r}\left[P_{\text {Supplier }(s r)}^{\text {reg }} C_{\text {Supplier }(s r)}\right] \\
+\sum_{\text {sa=1 }}^{S a}\left[P_{\text {Supplier }(s a)} C_{\text {Supplier }(s a)}\right]+\sum_{c=1}^{C}\left[P_{I D R(c)} C_{I D R(c)}\right]
\end{array}
$$

This function considers different resources such as DG units, consumers belonging to DR programs, and suppliers (both regular and additional). As already mentioned, regular or additional suppliers are used only in the case of aggregate production not complying with demand requests in order to have a network balance, as presented in Equation (2).

$$
\sum_{c=1}^{C}\left[P_{\operatorname{Load}(c)}^{\operatorname{Initial}}-P_{I D R(c)}\right]=\sum_{p=1}^{P} P_{D G(p)}+\sum_{s=1}^{S} P_{S u p(s)}+P_{N S P}
$$

For the network to flow safely and steadily, this equation is essential. Equation (2) shows that the sum of the possible reduction of each IDR consumer to its initial load should equal the sum of all DG units and suppliers ( $P_{\text {sup }}$ represents the regular and additional type). Still, in this equation, there is a variable that refers to the value of non-supplied power (NSP) - amount of load not fed by the production. It is intended that this value be kept null, proving that the network is being optimally managed.

Starting with the restriction associated with the consumers belonging to IDR, Equation (3), these are upper bound.

$$
P_{I D R(c)} \leq P_{I D R(c)}^{\operatorname{Max}}, \quad \forall_{c} \in\{1, \ldots, C\}
$$

For distributed resources, the DG units are limited by Equations (4)-(6). The case of wind power was applied to Equation (7) to realize the amount of available power where the value of cut-in and cut-out is considered. Considering that dispatchable generation can be controlled by operators, namely with Equation (4) and (5), the authors wanted to apply a power bound for the wind power. Through cut-in and cut-out speed, it is possible to know when the turbine blades begin to rotate and when they are brought to rest in order to avoid damages. In this way, with the Equation (7) and with all the parameters, namely the wind speed for the studied period, it is possible to find the right value to bound the wind power. Having this value, Equations (4) and (5) can be applied to this type of technology.

$$
\begin{gathered}
P_{D G(p)} \leq P_{D G(p)^{\prime}}^{M a x} \quad \forall p \in\{1, \ldots, P\} \\
P_{D G(p)} \geq P_{D G(p)^{\prime}}^{M i n} \quad \forall p \in\{1, \ldots, P\} \\
\sum_{p=1}^{P} P_{D G(p)} \leq P_{D G}^{\text {TotalMax }}, \\
P_{D G}^{\text {Wind }}=\frac{1}{2} \rho_{\text {air }} \pi r^{2} v^{3} C_{P}
\end{gathered}
$$

The technical limits for external suppliers are presented in Equations (9)-(11). Basically, in addition to being limited superiorly, the total amount that each type of external suppliers can make available is also limited.

$$
\begin{gathered}
P_{\text {Supplier(sr) }}^{\text {reg }} \leq P_{\text {Supplier }(s r)}^{\text {reg Max }}, \quad \forall s r \in\{1, \ldots, \text { Sr }\} \\
\sum_{\text {sr }=1}^{\text {Sr }} P_{\text {Supplier }(s r)}^{\text {reg }} \leq P_{\text {Supplier }}^{\text {reg Total }} \\
P_{\text {Supplier(sa) }}^{\text {add }} \leq P_{\text {Supplier }(s a)^{\prime}}^{\text {addMax }} \quad \forall s a \in\{1, \ldots, \text { Sa }\}
\end{gathered}
$$




$$
\sum_{\text {sa=1 }}^{\text {Sa }} P_{\text {Supplier }(s a)}^{a d d} \leq P_{\text {Supplier }}^{\text {add Total }}
$$

The last two equations, (12) and (13), limit the contribution of DG and IDR. This will allow the VPP to have control over them in an operating context or other limitations. Thus, the $\alpha$ parameter is created, where $\alpha_{D G}$ and $\alpha_{I D R}$ limit the DG units and DR consumers, respectively. This parameter has a value between 0 and 1 .

$$
\begin{aligned}
& \frac{\sum_{p=1}^{P} P_{D G(p)}}{\sum_{s r=1}^{S r}\left[P_{\text {Supplier }(s r)}^{\text {reg }}\right]+\sum_{s a=1}^{S a}\left[P_{\text {Supplier }(s a)}^{a d d}\right]+\sum_{p=1}^{P} P_{D G(p)}+\sum_{c=1}^{C}\left[P_{I D R(c)}\right]+P_{N S P}} \leq \alpha_{D G} \\
& \frac{\sum_{c=1}^{C} P_{I D R(c)}}{\sum_{s r=1}^{S r}\left[P_{\text {Supplier (sr })}^{\text {reg }}\right]+\sum_{s a=1}^{S a}\left[P_{\text {Supplier }(s a)}^{\text {add }}\right]+\sum_{p=1}^{P} P_{D G(p)}+\sum_{c=1}^{C}\left[P_{I D R(c)}\right]+P_{N S P}} \leq \alpha_{I D R}
\end{aligned}
$$

There are a total of two Equations ((2) and (7)) and five inequalities ((6), (9), (11), (12), and (13)), and the number of bounding constraints depends on the number of consumers, producers, or suppliers. Thus, regarding Equation (3), there will be as many as the number of consumers. In the case of Equations (4) and (5), there will be as many as the number of DG units. For Equations (8) and (10), there will be as many as the number of regular and additional suppliers. The variables and parameters used are real and not negative.

For example, considering that $\alpha_{I D R}$ has a value of 0.15 , this means that the contribution of all consumers belonging to DR programs should reduce their consumption to a value less than or equal to $15 \%$.

The values resulting from the optimization were used as input to the third phase of the methodology: aggregation. Given that and because the proposed methodology allows different time frames, a large database may exist, allowing better results to be obtained. In this context, the authors propose using clustering methods to form groups of resources. A well-known and used method of partitioning clustering was chosen-k-means. The algorithm of this method consists of finding the centroid value that represents each group. This value is found when the distance between this element and the remaining is minimal. To find this distance, several functions can be used, including Euclidean distance. The database is organized in matrix form, where the columns correspond to the different periods and the lines to the different resources under study. As a result, the VPP obtains the number of the group to which each object belongs, as well as the centroid of each group.

The aggregation has another value as input data-the number of clusters $\mathrm{k}$ to be formed. However, before the formation of groups, the optimal value of groups to which the database can be apportioned is necessary. There are methods that can find this value. Defining a set of $k$ clusters to be studied, methods like the elbow method and silhouette method can determine the optimum number of clusters- $\mathrm{k}_{\mathrm{opt}}$. Being a visual method, the elbow method is one of the oldest in use. Starting at 2 and increasing this value with a step 1, the total within-cluster sum of square (wss) is calculated for each of them. Reaching a value where the cost drastically reduces and then hits the plateau, the $\mathrm{k}_{\mathrm{opt}}$ was found. In other words, by plotting the results of the study set, it will be possible to find an "elbow" considered as an indicator of the appropriate number of clusters. For the other method referred to, the silhouette method, the silhouette value is calculated for each point - this value is measured by resemblance between one point and the remainder of the same group when compared with other clusters. To compare the set of $\mathrm{k}$ studied, the average silhouette width (ASW) is calculated for each cluster and the highest value is considered the $\mathrm{k}_{\mathrm{opt}}$ from that database. 
The remuneration phase is used as a motivation for the continuous participation in the management of the market. As mentioned above, the formation of the remuneration tariff is made through the sum of two variables: fixed tariff included in the contract with the aggregator and the indexed tariff that depends on the market price. The weight that each has in the final tariff depends on the VPP and is willing to increase or decrease its risk. Considering the remuneration groups, the tariff for each of them is found through the maximum of the tariffs of the consumers of each group. This amount is then applied as compensation to all those belonging to said group.

A new phase is added in relation to previous work done by the authors, [3] —-the classification phase. It is considered that the phases explained so far will be used in a planning approach. Although, in the case of real-time operation, it will be necessary to find a solution that is feasible in a timely manner. Thus, it is proposed to use a classification method to assist in this task, namely decision trees. With the results from the optimization, as shown in Figure 1, and using a decision tree, the VPP can assign each resource to a certain remuneration group (with a remuneration tariff) skipping tasks from other phases.

The proposed methodology was implemented in R software, which was used to run the presented case studies. For the first phase, the authors used the package lpsolve to find the solution for the optimization scheduling. In the aggregation phase, the authors opt for the package cluster.

\section{Case Study}

The proposed methodology was studied through a $30 \mathrm{kV}$ Real Portuguese distribution network, fed by a substation of $(60 / 30 \mathrm{kV})$ with a maximum capacity of 90 MVA. The database containing consumer profiles and DG units was collected for a full week as there are three-time frames to be studied-whole week (WW), weekend (W), and week days (WD). In addition, the database is divided into periods of $15 \mathrm{~min}$; thus, in this case study, the optimization phase is applied for each $15 \mathrm{~min}$. Thus, for WW, there are data for 672 periods; for W, there are for 192; and for WD, there are for 480.

This case study contains a total of 548 DG units, with seven different types of technologies: small hydro, waste-to-energy, wind, photovoltaic, biomass, fuel cell, and co-generation. Table 1 presents the characteristics of these units, namely, the value of tariffs, capacity, and total number of each type.

Table 1. Distributed generation units' characterization.

\begin{tabular}{|c|c|c|}
\hline Type & Tariff [m.u./kWh] & \# Units \\
\hline Biomass & 0.1206 & 25 \\
\hline Co-generation & 0.0975 & 16 \\
\hline Fuel Cell & 0.0945 & 13 \\
\hline Photovoltaic & 0.2889 & 208 \\
\hline Small Hydro & 0.0961 & 25 \\
\hline Waste-to-Energy & 0.0900 & 7 \\
\hline Wind & 0.0988 & 254 \\
\hline \multicolumn{2}{|c|}{ Total } & 548 \\
\hline
\end{tabular}

For consumers, there are five different types and their characteristics are presented in Table 2. This table contains the type, number, and tariffs to be applied. The tariffs are divided according to a schedule, and there are three types of schedules. This division is used by Portuguese energy traders. With this, single-tariff is when the tariff is the same all day; double-tariff when the day is divided into off-valley periods and valley periods; triple-tariff when there are peak, off-peak, and valley periods. Tariffs are different depending on the day of the week and season. The authors assumed that domestic and small commerce consumers exercised a single-tariff contract; medium commerce consumers a double-tariff contract; and large commerce and industrial would opt for a triple-tariff contract. The week studied is between 1 and 7 February 2019. Thus, the tariffs presented are the winter tariffs. 
Table 2. Consumers' characterization.

\begin{tabular}{ccccc}
\hline Type of Consumer & Type of Schedule & Period & $\begin{array}{c}\text { Tariff } \\
{[\mathbf{m} . \mathbf{u} . / \mathbf{k W h}]}\end{array}$ & \# Units \\
\hline Domestic (DM) & Single $\leq 2.3 \mathrm{kVA}$ & - & 0.1426 & 10,168 \\
Small Commerce (SC) & Single $\geq 2.3 \mathrm{kVA}$ & - & 0.1652 & 9828 \\
Medium Commerce (MC) & Double & Off-valley hours & 0.1948 & 82 \\
& & Valley hours & 0.1016 & \\
Large Commerce (LC) & Triple & Peak hours & 0.2253 & 85 \\
Industrial (ID) & & Off-valley & 0.1765 & 147 \\
& & Valley hours & 0.1016 & 20,310 \\
\hline
\end{tabular}

The aggregator is thus responsible for providing all the above characteristics, for each of the resources, in order to obtain viable results through this methodology. In the aggregation phase, the focus will be on consumers. With the three different time frames defined, it will be possible to perceive the difference of the remuneration for each one of them. The indexed tariffs used for the remuneration can be found in the historical data from OMIE (Operador del Mercado Ibérico de Energía_Polo Español) [22].

\section{Results}

Throughout this section will be presented the results obtained for the week chosen in the presented case study. This section is divided into subsections and according to the phases of the methodology considering that the first phase, creation of the scenario, has already been treated in the previous section. Thus, the first subsection deals with optimization results. The results will be presented to both consumers (value reduced from their consumption) and producers (the power used from each technology). These results are presented for the whole week-672 periods.

In the worst case, WW, the problem presents about 20,899 non-negative variables for the 672 periods, resulting in a matrix with 14,044,128 elements. There are 21,446 bounding constraints, 6 inequality constrains, and 2 equality constraints for each period studied in this case study. The running time for each period was approximately $110 \mathrm{~s}$, on a PC with $2.10 \mathrm{GHz}$ Intel ${ }^{\circledR}$ Xeon ${ }^{\circledR}$ CPU E5-2620 and 16.0GB RAM.

Regarding the aggregation phase, the results of the study are presented only for the optimum number of clusters for the input database, also found in this section. It is recalled that this complete study will be done for the three scenarios-WW, WD, and W. In the remuneration, the formation of the tariff is presented as well as the comparison of different methods for the calculation of the final remuneration. The classification phase tests the performance of the decision tree for each scenario in order to find the appropriate groups for a new consumer considering the optimization results.

\subsection{Optimization}

Figure 2 shows the results obtained for the DG units associated with the VPP in this case study. All these values are the sum of each type of resource, given that too many elements exist to present the results individually. When analysing the graph, it is evident the use, over all periods, of the co-generation, as well as fuel cell in large quantity, represents a large part of the production. Small hydro, biomass, and waste-to-energy units are also used throughout the week, however, their values are very low relative to the previous. Moving to the wind, considering Equation (6) and cut-in and cut-out velocity values, the results from this technology may be more limited. However, it turns out that when this technology is used, the value of fuel cell falls (visible, for example, between period 97 and 193). Regarding photovoltaic, although it is an intermittent technology, it is used whenever possible. Although co-generation technology was active during the entire study period, the highest sum of production was achieved by wind units, reaching close to $8 \mathrm{MW}$ in several periods. This result can be justified by the difference in the number of existing units of these two technologies in this case 
study. The maximum sum reached in one of the study periods was $7919.83 \mathrm{~kW}$ through wind, followed by co-generation with $6910.10 \mathrm{~kW}$.

Figure 3 shows the sum of the results obtained for each type of consumers considered in this case study compared with the initial load. Consumers of medium commerce are the ones who have achieved, in general, higher values of reduction of their consumption through DR programs. Next are the domestic type consumers. However, the difference between the number of consumers in each type is as follows: domestic has 10,168 elements and medium commerce has only 85 elements. The highest reduction sum obtained in this study for the 672 periods was through the medium commerce type, reaching $5627.38 \mathrm{~kW}$. The other types with higher values, domestic and small commerce consumers, obtained $4684.68 \mathrm{~kW}$ and $3858.34 \mathrm{~kW}$, respectively.

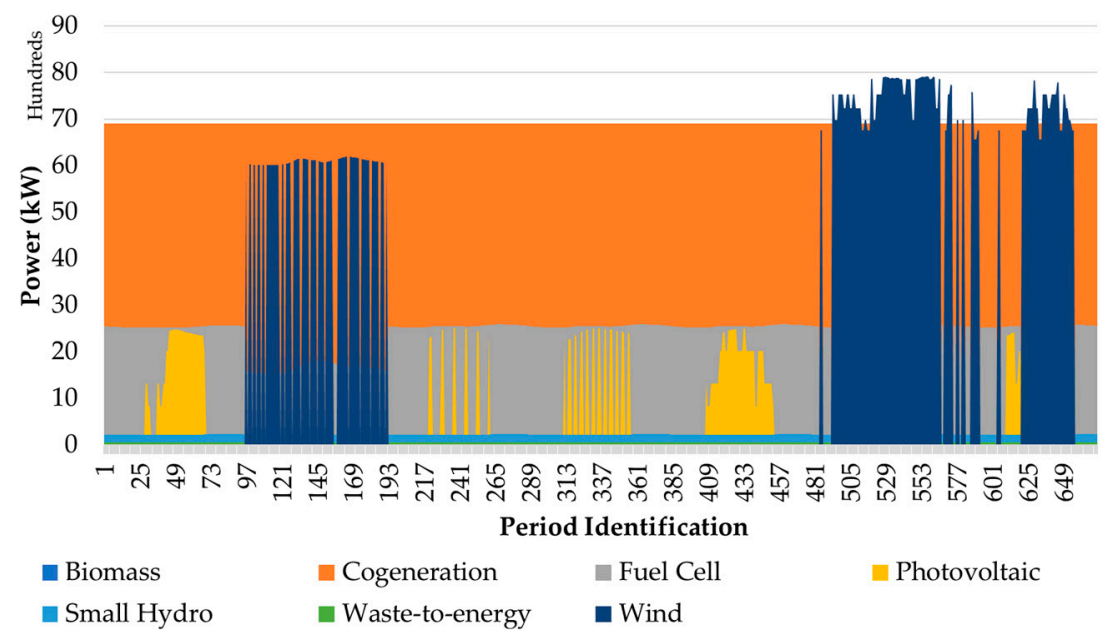

Figure 2. Results from optimization for the different DG units-sum of all units from each type.

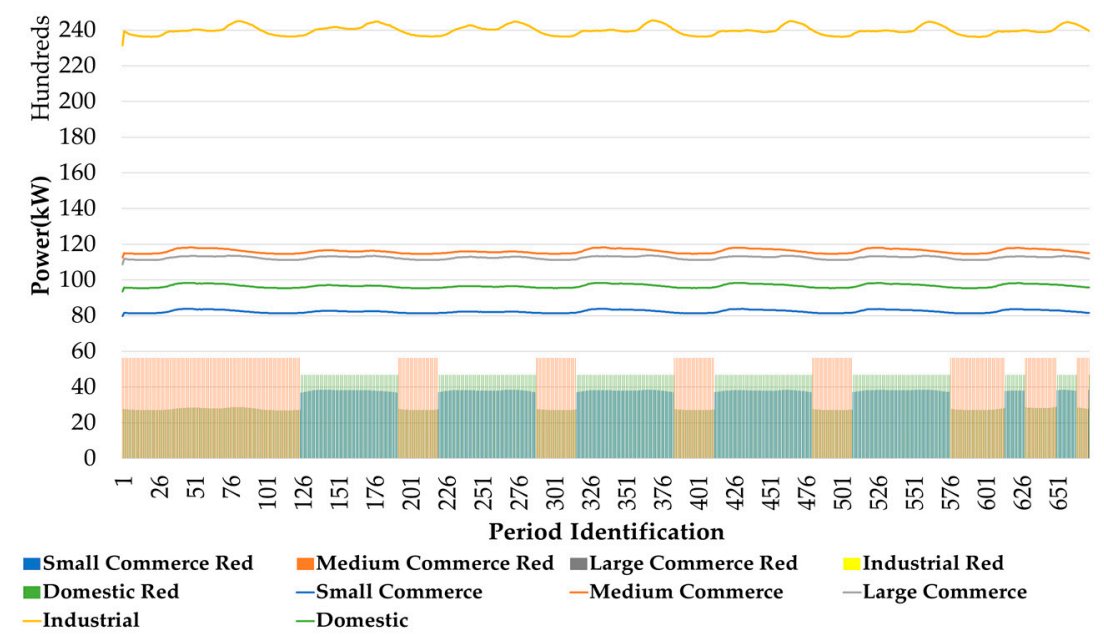

Figure 3. Results from optimization for the different types of consumers-sum of all consumers from each type (in red) and the initial load for comparison.

\subsection{Aggregation}

In this phase of aggregation, the authors propose finding the optimal value of groups to form the database used as input to the chosen clustering method. The following subsection, optimum number of clusters $\mathrm{k}$, presents the results for two methods that are commonly used to solve this problem. In the second subsection, groups formation, the results obtained using the k-means method are presented for the $\mathrm{k}_{\mathrm{opt}}$ considered. The composition of each group is presented as well as the centroids found in each group for each of the scenarios being studied. 
Optimum number of clusters- $\mathrm{k}_{\mathrm{opt}}$

Figure 4 presents the results for the elbow and silhouette methods. These methods were studied for a set of $\mathrm{k}$-between 2 and 15.

As already mentioned, the elbow method intends to evaluate in which $\mathrm{k}$ is that the value of wss reaches a plateau, that is, when the difference between $\mathrm{k}$ and $\mathrm{k}+1$ is not so significant when compared with the rest. As this is a visual method, the objective is to find in the graph obtained with the results an "elbow". For the three scenarios studied, the authors consider that $\mathrm{k}=3$ is the optimal value.

Regarding the silhouette method, the objective is to find the maximum value within the ASW calculated for the set of $\mathrm{k}$ studied and, unlike the previous method, not being a visual method does not suffer from the errors that can come from the opinion of the analyst. Although, the maximum value of ASW was found in $\mathrm{k}=3$.

(a) Whole Week

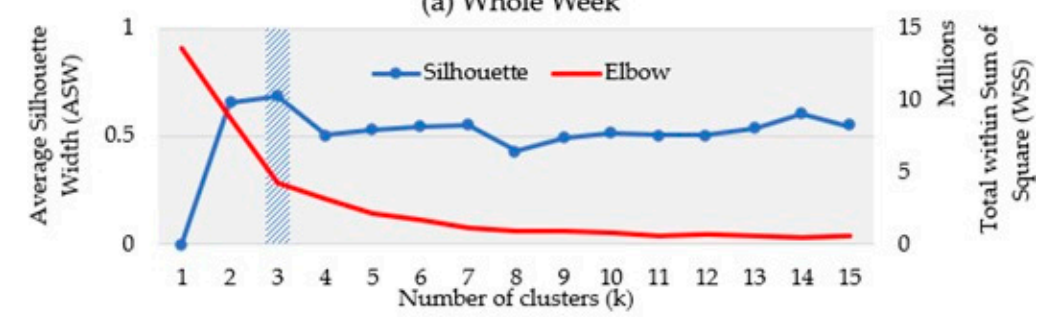

(b) Week Days

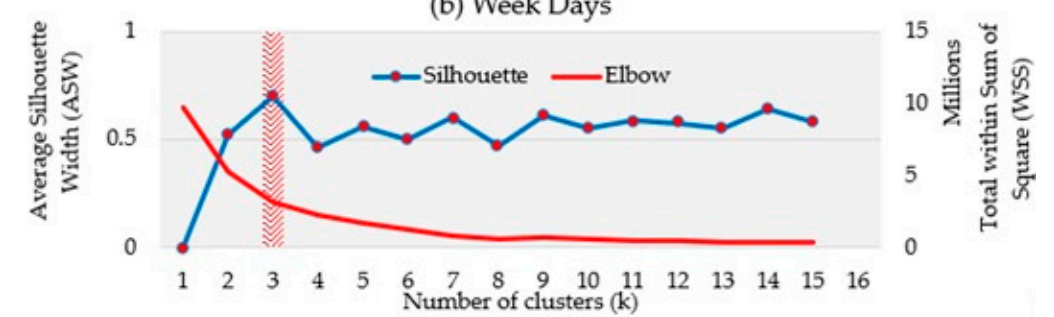

(c) Weekend

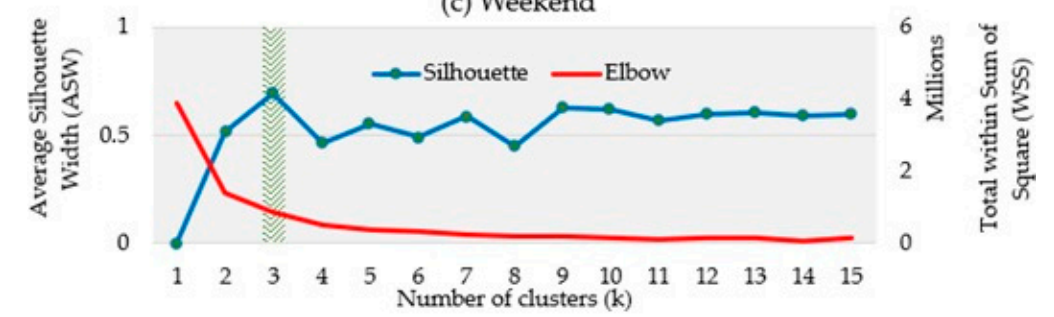

Figure 4. Elbow method vs. silhouette method. (a) Results for the whole week scenario; (b) results for the week days scenario; (c) results for the weekend scenario.

In this way, through the results obtained in the previous subsection, $\mathrm{k}_{\mathrm{opt}}=3$ was studied. Figure 5 shows the results obtained by applying the k-means method. The input data from this phase were the results obtained in the optimization for all types of consumers belonging to DR programs. This figure is divided according to the three scenarios studied and shows the number of elements and type of consumers that exist in each group.

Starting with the analysis of the first scenario, WW, Group 2 is composed only by elements of medium commerce, with 27 elements in total. Group 3, is composed only by domestic and small commerce elements, containing 2321 and 1146 elements, respectively. Moving on to Group 1, it contains all types of consumers, containing the elements of large commerce and industrial in its entirety. Furthermore, domestic contains 7847 elements, small commerce contains 8682 elements, and medium commerce contains 55 elements.

By analysing the WD scenario, the clustering method assigned the same number of elements of the groups when compared with the WW scenario. However, the identification of the group is different. Group 3 agglomerates of all types of consumers, with the number of elements being very similar to 
Group 1 of the WW scenario. Group 2 in WD is Group 2 in WW. For Group 1 in WD, this is Group 3 in WW.

However, the same situation does not occur with scenario $\mathrm{W}$. In this scenario, k-means assigns to Group 1 about 51 elements of medium commerce, 2321 elements of domestic, and 1146 elements of small commerce. For Group 3, 27 medium commerce elements were assigned. Finally, Group 2 agglomerates, once again, all types of consumers: domestic are 7847 elements; small commerce are 8682 elements; medium commerce are 4 elements; large commerce are 85, and industrial are 147 elements.

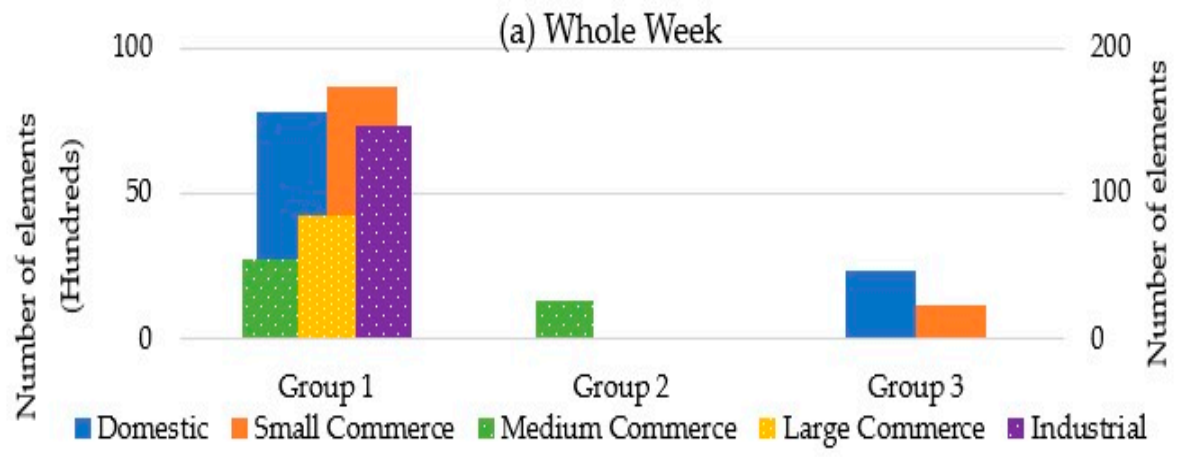

100

(b) Week Days

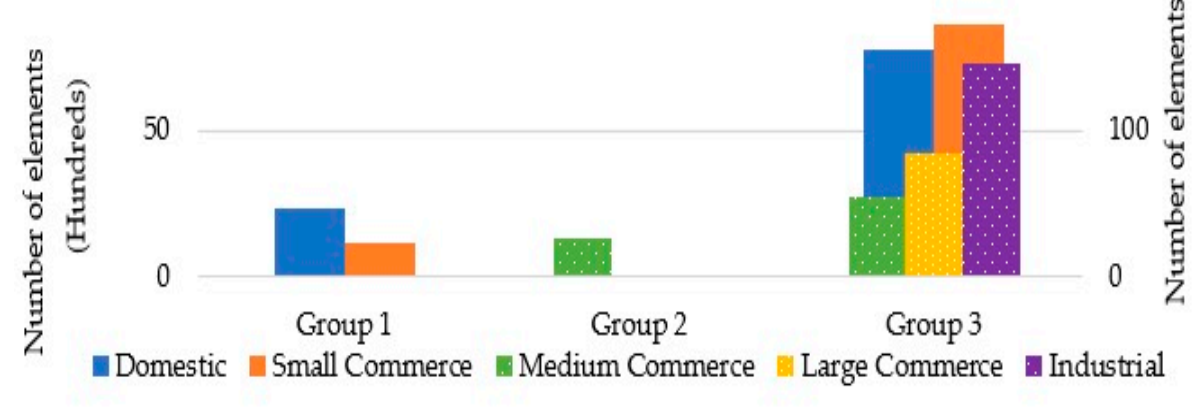

100

(c) Weekend

200

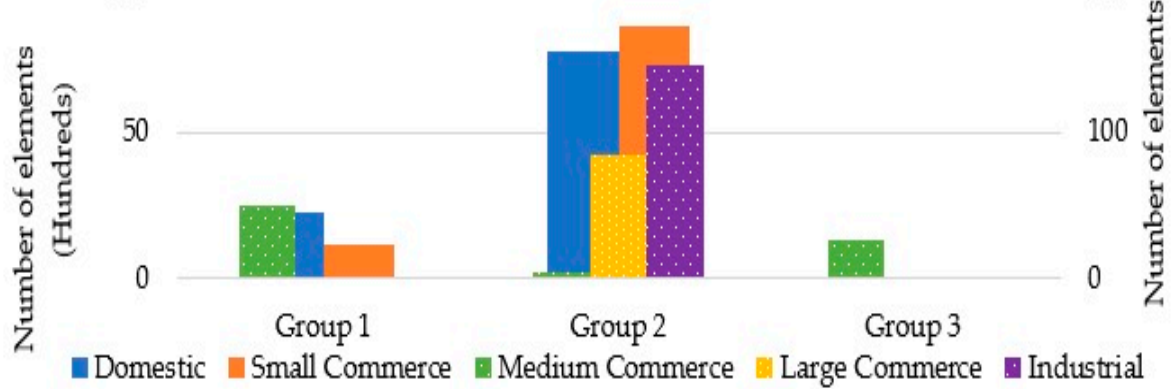

Figure 5. k-means method. (a) Results for the whole week scenario; (b) results for the week days scenario; (c) results for the weekend scenario.

Remembering the effect that the formation of these groups will have on final remuneration, as can be seen, the groups contain different types of consumers. The tariffs are different and, sometimes, higher. With this, it will be possible to remunerate them according to their performance, and thus in a fairer way. Through the approach proposed by the authors, the formation of a group tariff may have a more positive impact in order to influence the consumer to participate in this type of program.

Figure 6 shows one of the results obtained as output of the clustering method-the centroid value. The centroid value allows the VPP to obtain the average reduced power in each group. Because of the difference of scales between groups, the graphs in Figure 6 have two axes. The curves shown for the secondary y-axis, shown to the right of the figure, are indicated by a green dotted line. 


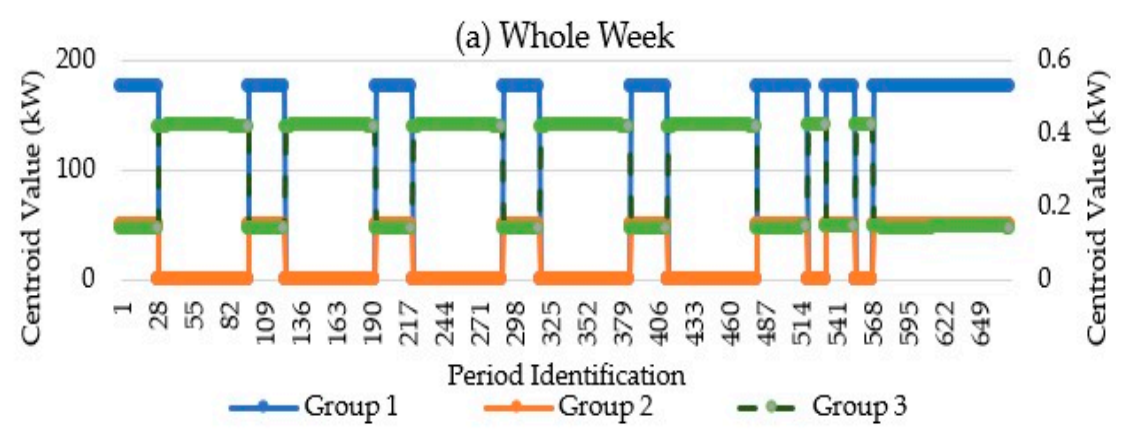

(b) Week Days
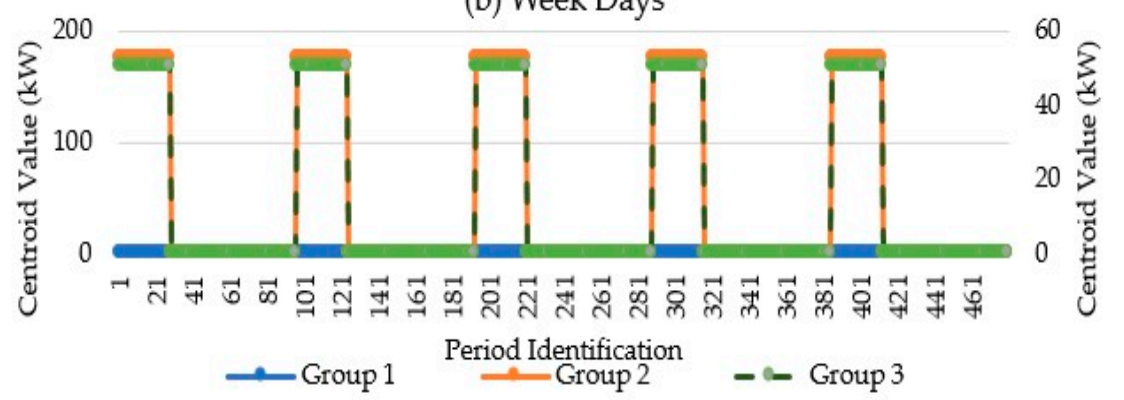

(c) Weekend

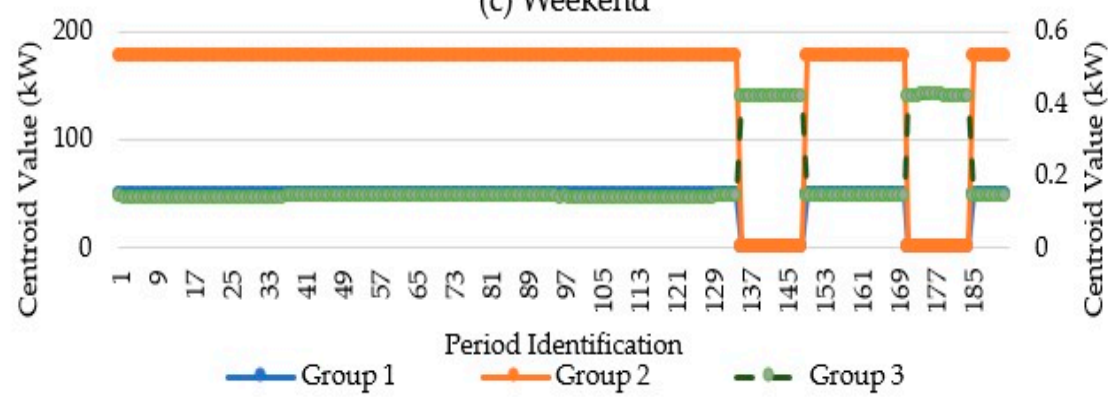

Figure 6. Centroid value. (a) Results for the whole week scenario; (b) results for the week days scenario; (c) results for the weekend scenario.

Although the clustering method has associated different groups, after the analysis of Figure 6, we can conclude there are three bands for reduction: high, medium, and low. In this way, there are two groups of consumers that do not reduce more than $\sim 1 \mathrm{~kW}$; a group that has average reductions of approximately $50 \mathrm{~kW}$ and the highest reduction values reach values above $175 \mathrm{~kW}$. With this information, the VPP can understand the behaviours of the consumers and the tendency of reduction.

\subsection{Remuneration}

At this stage, the remuneration groups were already formed as foreseen in the model proposed by the authors. Thus, in the subsection, remuneration tariff, the formation of the remuneration tariff is presented, such as the values from the indexed tariff for the period studied. Regarding the subsection, final remuneration, intending to prove the feasibility of the methodology for reducing the costs of the VPP, the authors compared several remuneration methods with the proposal in the presented model.

\subsubsection{Remuneration Tariff}

The authors propose that the remuneration tariff should be formed considering a fixed rate and an indexed rate. As already mentioned, this indexed tariff will add a share of uncertainty to this value, as it will depend on the market value. Table 3 presents the average price values for each of the days of the week studied, between 1 and 7 February 2019. These values were obtained through OMIE Iberian market. 
Table 3. Market price for the study period.

\begin{tabular}{cccccccc}
\hline & Feb, 1 & Feb, 2 & Feb, 3 & Feb, 4 & Feb, 5 & Feb, 6 & Feb, 7 \\
\hline Tariff [m.u./kWh] & 0.05268 & 0.03846 & 0.04927 & 0.06122 & 0.06166 & 0.06140 & 0.05951 \\
\hline
\end{tabular}

Equations (14) and (15) show the way the authors considered to find the final remuneration rate of each group.

$$
\begin{gathered}
\text { FinalTariff }_{g}=\operatorname{perc}_{f i x} \times \operatorname{Tariffix}_{g}+\operatorname{perc}_{I N D} \times \operatorname{TariffIND} \\
\operatorname{perc}_{f i x}+\operatorname{perc}_{I N D}=1
\end{gathered}
$$

The weight of each of the variables is decided by the VPP, and the authors have already done a study in this area in order to understand the influence of this weight on the final remuneration [3]. For this case study, it is assumed that each tariff would have a weight of $50 \%$ in the formation of the remuneration rate.

\subsubsection{Final Remuneration}

The proposal will be to test the remuneration through the different methods presented in Table 4 . There are four methods. Only method 1 is not applied according to the group that was obtained in the previous phase.

Table 4. Methods' definition.

\begin{tabular}{ccl}
\hline Method & Type of Remuneration & \multicolumn{1}{c}{ Description } \\
\hline 1 & Individual & $\begin{array}{l}\text { Each consumer receives according to the contracted tariff (fix tariff) } \\
\text { Each consumer receives according to the average of the tariffs of } \\
\text { the group that belongs (fix tariff) } \\
\text { Each consumer receives according to the maximum of the tariffs of } \\
\text { the group that belongs (fix tariff) } \\
\text { Each consumer receives according to the average of the tariffs of } \\
\text { the group that belongs. The tariff is formed through the sum of fix } \\
\text { tariff and index tariff }\end{array}$ \\
\hline
\end{tabular}

The proposal for method 1 will be to remunerate each individual consumer. Many of the works of the literature opt for this method. Therefore, according to the reduced value for a given period, the consumer is remunerated according to the tariff applied in that period. The remaining methods were applied to the three groups found through the aggregation phase. Therefore, method 2 applies the mean and method 3 applies the maximum. For method 4, the maximum is applied, but for the tariff formed in the previous subsection, considering the fixed tariff and the indexed tariff. Table 5 presents the results for the four methods presented.

Table 5. Remuneration Methods.

\begin{tabular}{cccc}
\hline Method & Whole Week [m.u] & Weekend [m.u] & Week Days [m.u] \\
\hline 1 & $885,496.59$ & $195,349.72$ & $690,146.86$ \\
2 & $880,536.12$ & $288,875.35$ & $646,503.79$ \\
3 & $1,278,258.91$ & $309,979.03$ & $802,682.48$ \\
4 & $794,850.47$ & 190483.39 & $521,568.37$ \\
\hline
\end{tabular}

After analysing the table, itis easily perceived that method 4 gets the lowest values for all three scenarios. Analysing scenario by scenario, for WW, the highest value of remuneration was generated through method 3. The difference between the other methods is significant, with the lowest value of remuneration being $794,850.47$ m.u. for method 4 compared with 1,278,258.91 m.u. for method 3 . 
In the case of $\mathrm{W}$, although the highest remuneration value continues to be generated by method 3 , the difference for the other methods, namely method 2, is not as high. However, method 4 maintains the position, obtaining the lowest compensation value. So far, method 1 had obtained values lower than method 2, but was different in WD, obtaining 690,146.86 m.u. and 646,503.76 m.u., respectively. Method 3 reaches $802,682.48$ m.u., again being the highest value.

Through this analysis, it was proven that the method that the authors considered in the model presented in this paper is able to achieve the proposed objective-lower costs for VPP considering the uncertainty of the market prices while still remunerating consumers fairly.

\subsection{Classification}

The classification phase was added to this model for this paper regarding other works carried out by the authors. The authors consider that, for a case of operation, it will be advantageous to use a classification method, namely the decision tree, in order to assign to a given consumer a group according to the results obtained in the optimization. This is a novelty regarding previous works. The decision trees obtained are presented in Figures 7-9. This classification method has as input the optimization results for the week studied throughout this paper. In fact, the methodology gives the VPP the freedom to use whatever database in terms of size and variety. The goal of this new phase is to, with the classification method, assign a remuneration group to all elements according, for example, their value of reduction (in case of the consumers). Figure 7 presents the results from the WW scenario.

As for the method used for the creation of the remuneration groups used all the results of the WW scenario, studying its pattern of consumption for the full week, Group 1 is divided into two bands: less than $0.66 \mathrm{~kW}$ and between 5.7 and $64 \mathrm{~kW}$. In Group 2 are the larger reductions, being greater than $64 \mathrm{~kW}$. The elements of Group 3 are between $0.66 \mathrm{~kW}$ and $5.7 \mathrm{~kW}$. Figure 8 shows the results for the WD scenario.

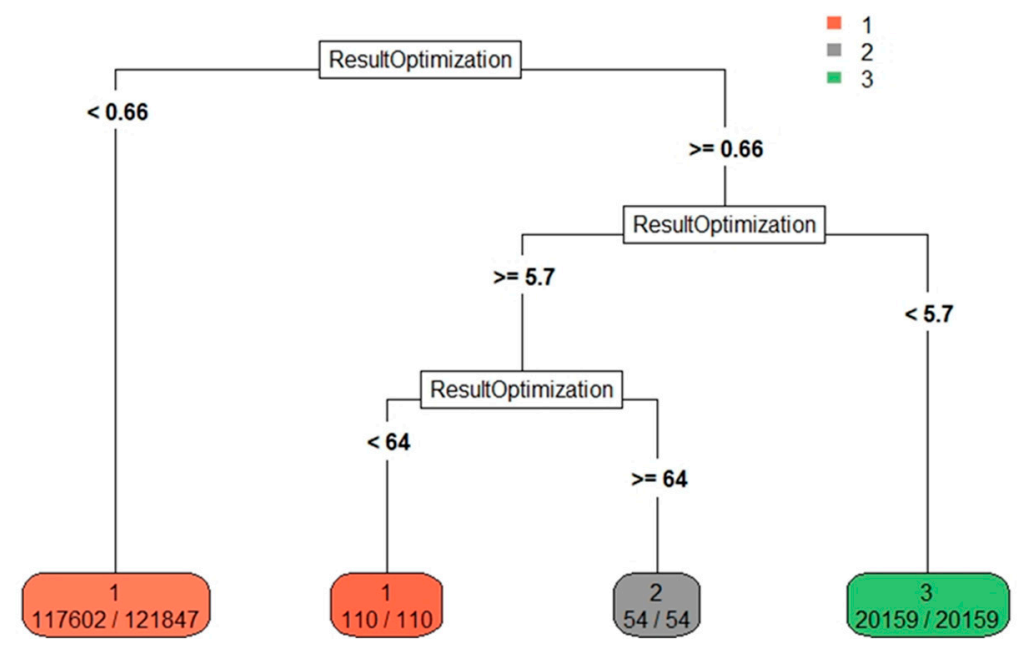

Figure 7. Decision tree. Results for the whole week scenario.

The same situation occurs for the WD scenario, but this time with Group 3: elements with values lower than $0.66 \mathrm{~kW}$ and between 5.7 and $64 \mathrm{~kW}$. In Group 2 are larger reductions, being greater than $64 \mathrm{~kW}$. The elements of Group 3 are between $0.66 \mathrm{~kW}$ and $5.7 \mathrm{~kW}$. Figure 8 shows the results for the W scenario.

For the W scenario, presented in Figure 9, the groups are spread over more ranges of values. Group 1 includes values between 0.66 and $5.7 \mathrm{~kW}$ and values between 35 and $64 \mathrm{~kW}$. In relation to Group 2, there are values below $0.66 \mathrm{~kW}$ and between 5.7 and $35 \mathrm{~kW}$. Group 3 agglomerates elements with reductions greater than $64 \mathrm{~kW}$. With this decision tree, the authors support the task of the VPP in terms of operation, moving on to the final remuneration. 


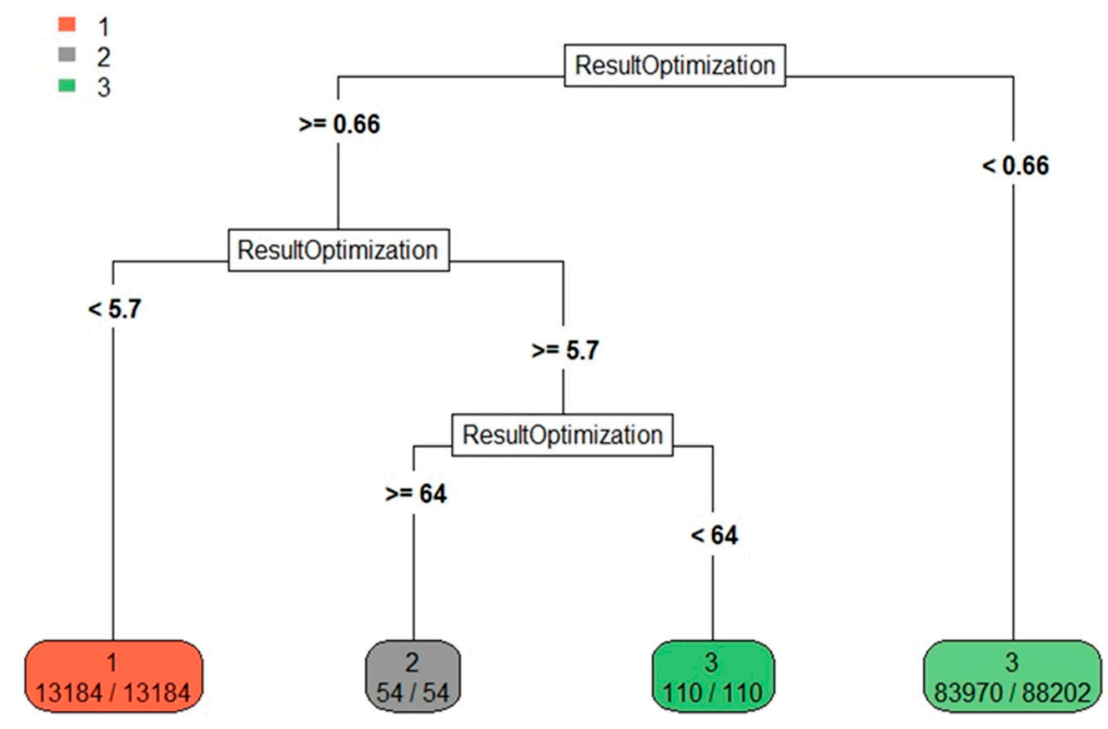

Figure 8. Decision tree. Results for the week days scenario.

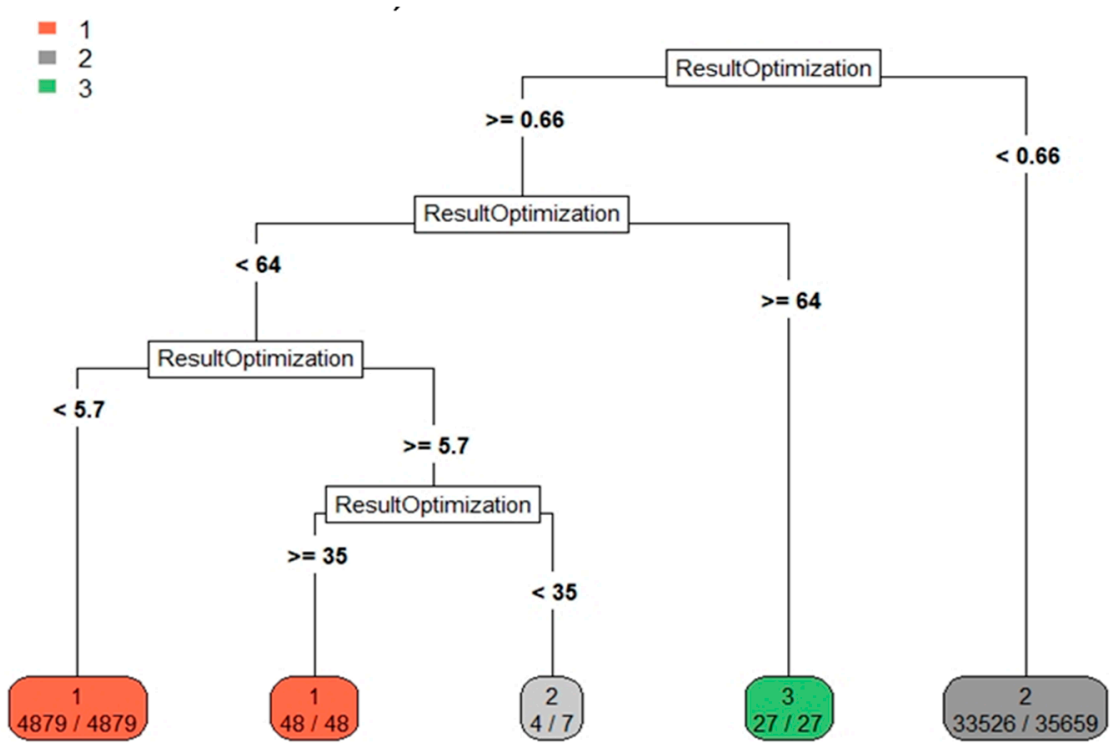

Figure 9. Decision tree. Results for the weekend scenario.

Method Application

In order to prove the viability of new phase introduced in the proposed methodology, a simulation with five new consumers was carried out. Comparing between the proposed path in the present paper, optimization and classification, and following all the phases from the proposed methodology. Table 6 show the characteristics from the new consumers. The present results were tested for a Wednesday at 11:00 in the morning. PinLoad represents the initial load at the moment and Pmaxidr the maximum amount that can be reduced according the DR program from each consumer.

Table 6. Characteristics from new consumers.

\begin{tabular}{cccccc}
\hline & \multicolumn{5}{c}{ Consumer } \\
\cline { 2 - 6 } Parameter & $\mathbf{1}$ & $\mathbf{2}$ & $\mathbf{3}$ & $\mathbf{4}$ & $\mathbf{5}$ \\
& $\mathbf{S C}$ & $\mathbf{D M}$ & $\mathbf{L C}$ & $\mathbf{S C}$ & $\mathbf{M C}$ \\
\hline PinLoad & 3.658 & 1.859 & 71.099 & 2.576 & 37.127 \\
\hline Pmaxidr & 1.747 & 0.887 & 34.871 & 1.230 & 17.729 \\
\hline
\end{tabular}


Performing the optimization phase, the results for one period are represented in Table 7.

Table 7. Optimization Results.

\begin{tabular}{cccccc}
\hline \multirow{2}{*}{ Results } & \multicolumn{5}{c}{ Consumer } \\
\cline { 2 - 6 } & $\mathbf{1}$ & $\mathbf{2}$ & $\mathbf{3}$ & $\mathbf{4}$ & $\mathbf{5}$ \\
& SC & DM & LC & SC & MC \\
\hline Reduction & 1.747 & 0.887 & 0 & 1.230 & 0 \\
\hline
\end{tabular}

Consumers 1,2, and 4 are requested to reduce the maximum value. On the contrary, the results from the optimization for Consumers 3 and 5 do not request any kind of reduction. Following all the phases from the proposed methodology, aggregation and remuneration were performed. The resulting groups, as well as the remuneration tariffs, are presented in Tables 8 and 9.

Table 8. Aggregation groups. WW, whole week, W weekend; WD, week days.

\begin{tabular}{cccccc}
\hline & \multicolumn{5}{c}{ Consumer } \\
\cline { 2 - 6 } Scenario & $\mathbf{1}$ & $\mathbf{2}$ & $\mathbf{3}$ & $\mathbf{4}$ & $\mathbf{5}$ \\
& SC & DM & LC & SC & MC \\
\hline W & 1 & 1 & 2 & 1 & 2 \\
WW & 3 & 3 & 1 & 3 & 1 \\
WD & 1 & 1 & 3 & 1 & 3 \\
\hline
\end{tabular}

Table 9. Remuneration tariffs.

\begin{tabular}{cccccc}
\hline & \multicolumn{5}{c}{ Consumer } \\
\cline { 2 - 6 } Scenario & $\mathbf{1}$ & $\mathbf{2}$ & $\mathbf{3}$ & $\mathbf{4}$ & $\mathbf{5}$ \\
& SC & $\mathbf{D M}$ & LC & SC & MC \\
\hline W & 0.1986 & 0.1986 & 0.1547 & 0.1986 & 0.1547 \\
WW & 0.1551 & 0.1551 & 0.1551 & 0.1551 & 0.1551 \\
WD & 0.1531 & 0.1531 & 0.1551 & 0.1531 & 0.1551 \\
\hline
\end{tabular}

The aggregation phase was performed with the selected clustering method, k-means, and the results are present only for the $k_{\text {opt }}$ chosen earlier. The case of the remuneration phase considered the method with lower costs for the VPP, but maintaining fair remuneration for the participants in the management of the market transactions.

For scenario W, Consumers 1, 2, and 4 went to the same group, Group 1, and consequently the same remuneration tariff was attributed, 0.1986 . In the case of large commerce and medium commerce consumers, these were clustered in Group 2 where the compensation tariff was 0.1547.

In the scenario WW, the selected clustering method groups Consumers 1,2, and 4, again, in the same group. However, rather than Group 1 as scenario W, the chosen group was Group 3. The tariff for this group is lower. Considering that WW gathers all the tariffs for the selected week, after finding the maximum for each group, the tariff was the same. In this way, when finding the value of the final tariff, the value of the tariff for all groups is 0.1551 . With this, although Consumers 3 and 5 went to a different group, Group 1, the compensation value was equal.

For the WD scenario, Group 1 aggregates Consumer 1, 2, and 4 and Group 3 with Consumer 3 and 5. The remuneration tariff was higher in Group 3, with 0.1551 , the same as in scenario WW, and Group 1, through method of remuneration 4, results in a tariff equal to 0.1531 . 
Returning to optimization phase, and following the path suggested in the present paper for real-time operation, a classification method, namely decision trees, was used to decided to which group each consumer should join. Figures 10 and 11 present the results from applying the decision trees created previously to the new consumers. The decision for the three different scenarios, WW, WD, and W, was presented, respectively. Each path has the color of the assigned group: Group 1 is red, Group 2 is grey, and Group 3 is green.
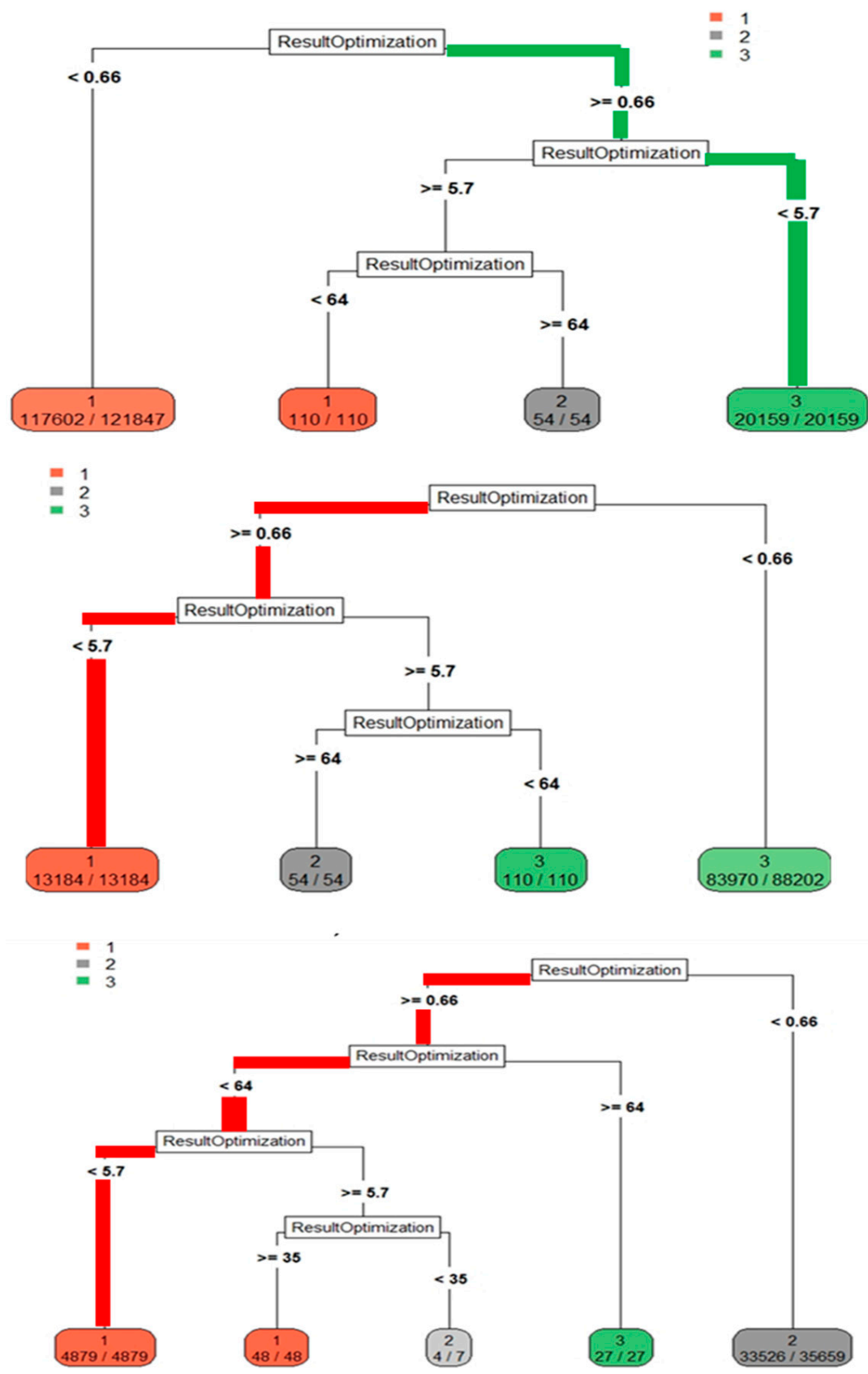

Figure 10. Decision tree. Results for Consumers 1, 2, and 4.

Figure 10a shows the result for scenario WW, where Consumers 1, 2, and 4 had a value of reduction superior than 0.66 , but inferior to 5.7, being assigned to Group 3 .

Figure $10 \mathrm{~b}$ presents the result for scenario WD, where the mentioned consumers followed the same path as scenario WW: value of reduction superior than 0.66 , but inferior to 5.7 , but this time being assigned to Group 3. 
Figure $10 \mathrm{c}$ demonstrates the result for scenario $\mathrm{W}$. The consumers had value of reduction superior than 0.66 , but inferior to 64 . However, the value was still inferior to 5.7, being assigned to Group 1 .

Figure 11a presents the result for scenario WW for the large and medium commerce consumers. As the value of reduction resulting from the optimization performed in the scheduling phase, Group 1 was assigned. The decision for the remaining scenarios is the same: inferior to 0.66 . For scenario WD, the group assigned was Group 3 and the result for scenario W was being assigned to Group 2. With these results, the viability of the proposed method was proven, being very useful for the VPP in real-time operation situations.
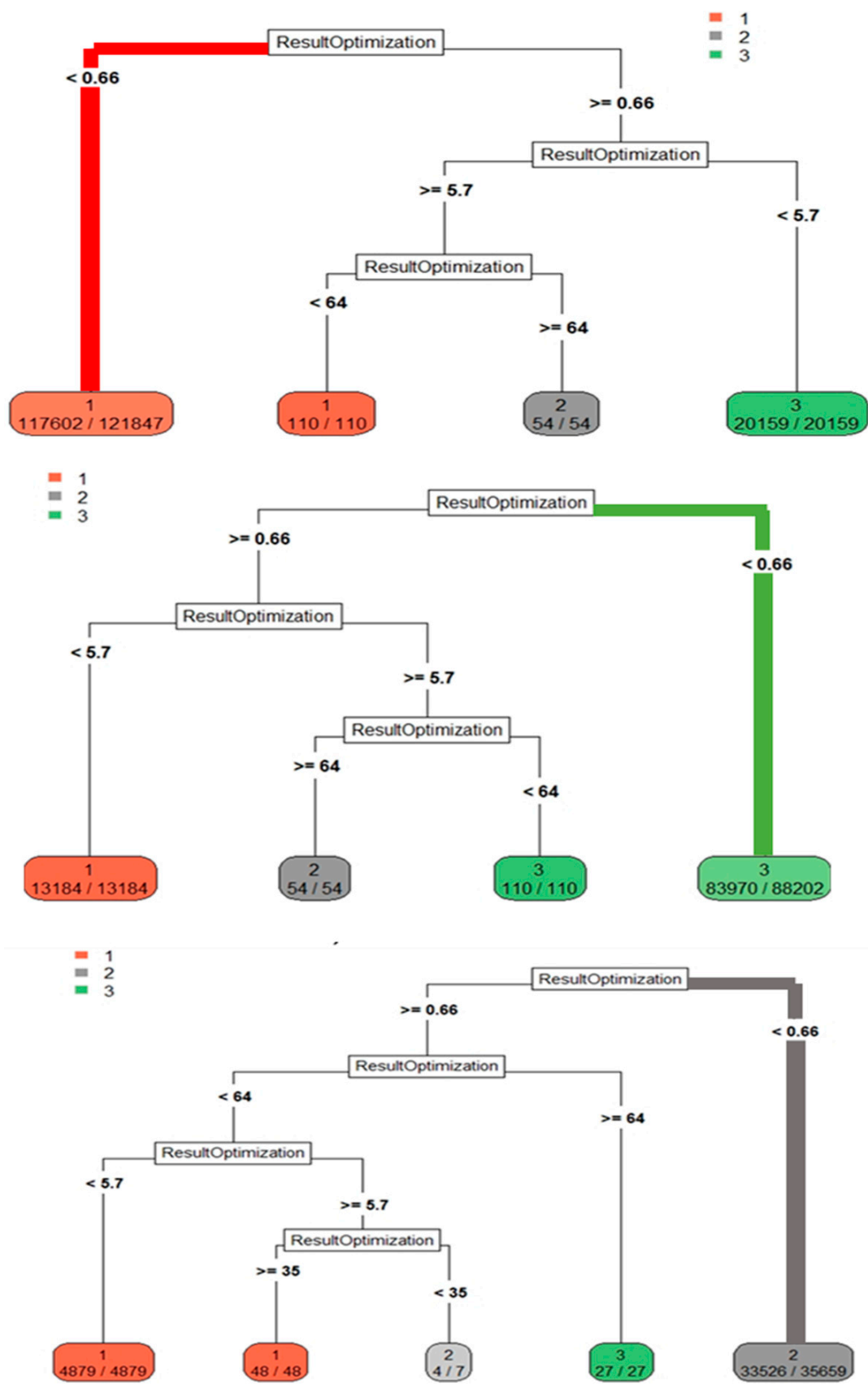

Figure 11. Decision tree. Results for Consumers 3 and 5.

\section{Conclusions}

Throughout this paper, a methodology was presented that proposes supporting VPP in the aggregation of small resources, until now, with little focus on current business models. The main 
objective is to remunerate these resources in a fair way, with the formation of remuneration groups being proposed by the authors. This approach benefits VPP with a large amount of energy to participate in the wholesale market and by minimizing operating costs. Being a resource in this model, in addition to being able to participate in the market transitions, will be remunerated in a fair way according to their participation in the management of the market.

As innovation from previous works, in this paper, the authors added a new phase, classification. The main goal is to give in proper time, considering that VPP will work in real time, the final remuneration group for each resource considering the results from optimal scheduling. In this way, the global goal of fair remuneration is still considered, and the methodology is improved.

Other business models presented in the literature opt for individual remuneration or by type of resource. The suggestion proposed by the authors uses clustering methods for the formation of groups, where the optimal number of groups is found for the studied database. Another innovation from this model is a multi-period approach, allowing the creation of a larger database for use in the clustering method. In this way, different tariffs will be tested—as the tariff is influenced by the schedule, making it possible to create a different remuneration tariff and possibly making it more beneficial for the resources, unlike the single period approach. In this paper, the authors proposed to select the maximum tariff for each group and remunerate each one of the members of this group with the same remuneration rate. It is recalled that the main objective will be to keep resources motivated to participate. By opting for a maximum rate, with the possibility of associating different resources (as aggregation depends on its contribution and not the type to which it belongs), it can act as an incentive. The formation of the tariff is one of the innovations, proposing the sum of two tariffs: fixed and indexed. The fixed part refers to time-of-use tariffs existing in Portugal. The indexed part of the final tariff adds some uncertainty as it depends on market prices. However, the VPP may choose the influence that this value will have on the remuneration tariff. It was proved that, when compared with other methods of remuneration, the method proposed by the authors obtains lower values of remuneration, being advantageous to the VPP, but still being able to pay the resources fairly.

The fact that this methodology includes several phases-scheduling, aggregation, and remuneration-in a single business model is a benefit for VPP. However, the authors go further and add a phase that may be quite useful in terms of operation. The first three phases can be used in the planning case. In the operation, it will be essential in time to realize in which group each resource is inserted. Thus, classification methods were used so that through the results obtained in the optimization, the VPP can link a resource to a remuneration group and to the compensation tariff without going through all the steps of the remaining phases. The study showed that according to the groups generated, the database was composed mainly by three types of reduction: small (below $6 \mathrm{~kW}$ ), medium (between 6 and $64 \mathrm{~kW}$ ), and high (above $64 \mathrm{~kW}$ ).

As future work, the authors want to further investigate the use of energy storage systems in the proposed model. As already mentioned, storage means are very advantageous in this area and will enrich and improve the proposed model. Another additional consideration for the proposed methodology could be aspects related to the electrical distribution network operation in the optimization phase, namely technical constraints such as the capacity of the electric lines or admissible voltage limits, including, in the methodology, even more factors that influence the normal operation of the network.

Author Contributions: Conceptualization, Z.V.; Data curation, C.S.; Formal analysis, C.S. and P.F.; Investigation, C.S. and P.F.; Methodology, P.F. and Z.V.; Resources, Z.V.; Visualization, C.S.; Writing-original draft, C.S.; Writing-review \& editing, P.F. and Z.V.

Funding: The present work was done and funded in the scope of the following projects: European Union's Horizon 2020 project DOMINOES (grant agreement No 771066), and UID/EEA/00760/2019 funded by FEDER Funds through COMPETE program and by National Funds through FCT.

Conflicts of Interest: The authors declare no conflict of interest. The founding sponsors had no role in the design of the study; in the collection, analyses, or interpretation of data; in the writing of the manuscript; and in the decision to publish the results. 


\section{Nomenclature}

\section{Indices}

$c$

$p$

sa

sr

Variables

$\mathrm{P}_{\text {DG }}(p)$

$\mathrm{P}_{\mathrm{IDR}}(c)$

$P_{\text {Supplier (sa) }}^{\text {add }}$

$P_{\text {Supplier(sr) }}^{\text {reg }}$

Parameters

$\alpha_{D G}$

$\alpha_{I D R}$

$\rho_{\text {air }}$

C

$C_{D G}(p)$

$C_{\text {IDR }}(c)$

$C_{\text {NSP }}$

Csupa (sa)

$C_{\text {Supr }}(s r)$

$\mathrm{C}_{p}$

FinalTariff $_{g}$

OC

P

PInitial $_{\text {Load }}(c)$

$\mathrm{P}_{\text {IDRmax }}(\mathrm{c})$

$P^{M a x}{ }_{D G}$

$\mathrm{P}_{\mathrm{NSP}}$

$P^{a d d \text { Max }}$ Sup (sa)

PaddTotal $_{\text {Sup }}$

Preg Max $_{\text {Sup }}$ (sr)

Preg Total $_{\text {Sup }}$

$P^{\text {TotalMax }} D G$

$\operatorname{perc}_{f i x}$

$\operatorname{perc}_{I N D}$

$r$

S

Tariffix

TariffIND

$v$

Acronyms

ASW

CHP

DG

DM

DR

ID

IDR

$\mathrm{k}_{\text {opt }}$

LC

$\mathrm{MC}$

NSP

OMIE

RES

$\mathrm{SC}$

VPP

W

WD

WSS

WW

Number of consumers $(c=1,2, \ldots, C)$

Number of generation units $(p=1,2, \ldots, \mathrm{P})$

Number of additional suppliers $(s a=1,2, \ldots, \mathrm{Sa})$

Number of regular suppliers $(s r=1,2, \ldots, \mathrm{Sr})$

Scheduled power for distributed generation unit $p$

Scheduled power reduction for Incentive-based demand response program for consumer $c$

Scheduled power for additional sa supplier

Scheduled power for a regular sr supplier

Usage limitation for distributed generation

Usage limitation for incentive-based demand response program

Air density $\left[\mathrm{kg} / \mathrm{m}^{3}\right]$

Maximum number of consumers c

Distributed generation unit $\mathrm{p}$ cost

Incentive-based demand response cost for consumer c

Non-supplied power cost

Regular sa supplier cost

Additional sr supplier cost

Power coefficient

Final remuneration tariff for each group g

Operation costs

Maximum number of producers $p$

Initial consumption of the consumers

Maximum scheduled power reduction for incentive-based demand response program for consumer $c$

Maximum power schedule in a distributed generation resource $\mathrm{p}$

Non-supplied power

Maximum power from an additional supplier sa

Maximum allowed total power from all the additional suppliers

Maximum power from a regular supplier sr

Maximum allowed total power from all the regular suppliers

Maximum allowed total power from all the distributed generation units

Percentage of fix tariff in the formation of the final remuneration tariff

Percentage of indexed tariff in the formation of the final remuneration tariff

Radius [m]

Maximum number of suppliers s

Remuneration tariff for each group $g$

Indexed tariff

Wind speed

Average silhouette width of a cluster

Combined heat and power

Distributed generation

Domestic

Demand response

Industrial

Incentive-based demand response

Optimum number of clusters

Large Commerce

Medium Commerce

Non-supplied power

Operador del Mercado Ibérico de Energía-Polo Español

Renewable energy Ssurce

Small Commerce

Virtual power player

Weekend

Week days

Total within-cluster sum of square

Whole week 


\section{References}

1. Shariatzadeh, F.; Mandal, P.; Srivastava, A.K. Demand response for sustainable energy systems: A review, application and implementation strategy. Renew. Sustain. Energy Rev. 2015, 45, 343-350. [CrossRef]

2. Bertoldi, P.; Zancanella, P.; Boza-Kiss, B. Demand Response Status in EU Member States; EUROPA: Luxembourg, LUXEMBOURG, 2016.

3. Silva, C.; Faria, P.; Vale, Z. Multi-Period Observation Clustering for Tariff Definition in a Weekly Basis Remuneration of Demand Response. Energies 2019, 12, 1248. [CrossRef]

4. Silva, C.; Faria, P.; Vale, Z. Discussing Different Clustering Methods for the Aggregation of Demand Response and Distributed Generation. In Proceedings of the 2018 IEEE Symposium Series on Computational Intelligence (SSCI), Bengaluru, India, 18-21 November 2018.

5. Silva, C.; Faria, P.; Vale, Z. Distributed Generation with Improved Remuneration. In Proceedings of the 2018 IEEE Symposium Series on Computational Intelligence, Bengaluru, India, 18-21 November 2018; Volume 217, pp. 1639-1644.

6. Wang, R.; Wang, P.; Xiao, G. A robust optimization approach for energy generation scheduling in microgrids. Energy Convers. Manag. 2015, 106, 597-607. [CrossRef]

7. Heydarian-Forushani, E.; Golshan, M.E.H.; Moghaddam, M.P.; Shafie-Khah, M.; Catalão, J.P.S. Robust scheduling of variable wind generation by coordination of bulk energy storages and demand response. Energy Convers. Manag. 2015, 106, 941-950. [CrossRef]

8. Nojavan, S.; Aalami, H.A. Stochastic energy procurement of large electricity consumer considering photovoltaic, wind-turbine, micro-turbines, energy storage system in the presence of demand response program. Energy Convers. Manag. 2015, 103, 1008-1018. [CrossRef]

9. Silva, C.; Faria, P.; Vale, Z. Clustering Support for an Aggregator in a Smart Grid Context. In Proceedings of the 18th International Conference on Hybrid Intelligent Systems (HIS), Porto, Portugal, 13-15 December 2018.

10. Jia, L.; Tong, L. Dynamic Pricing and Distributed Energy Management for Demand Response. IEEE Trans. Smart Grid 2016, 7, 1128-1136. [CrossRef]

11. Wei, W.; Liu, F.; Mei, S. Energy Pricing and Dispatch for Smart Grid Retailers Under Demand Response and Market Price Uncertainty. IEEE Trans. Smart Grid 2015, 6, 1364-1374. [CrossRef]

12. Mnatsakanyan, A.; Kennedy, S.W. A Novel Demand Response Model with an Application for a Virtual Power Plant. IEEE Trans. Smart Grid 2015, 6, 230-237. [CrossRef]

13. Ma, J.; Deng, J.; Song, L.; Han, Z. Incentive Mechanism for Demand Side Management in Smart Grid Using Auction. IEEE Trans. Smart Grid 2014, 5, 1379-1388. [CrossRef]

14. Acuña, L.G.; Ríos, D.R.; Arboleda, C.P.; Ponzón, E.G. Cooperation model in the electricity energy market using bi-level optimization and Shapley value. Oper. Res. Perspect. 2018, 5, 161-168. [CrossRef]

15. Carli, R.; Dotoli, M. Cooperative Distributed Control for the Energy Scheduling of Smart Homes with Shared Energy Storage and Renewable Energy Source. IFAC-PapersOnLine 2017, 50, 8867-8872. [CrossRef]

16. Carli, R.; Dotoli, M. Energy scheduling of a smart home under nonlinear pricing. In Proceedings of the 53rd IEEE Conference on Decision and Control, Los Angeles, CA, USA, 15-17 December 2014; pp. 5648-5653.

17. Sperstad, I.; Korpås, M.; Sperstad, I.B.; Korpås, M. Energy Storage Scheduling in Distribution Systems Considering Wind and Photovoltaic Generation Uncertainties. Energies 2019, 12, 1231. [CrossRef]

18. Hosseini, S.M.; Carli, R.; Dotoli, M. Model Predictive Control for Real-Time Residential Energy Scheduling under Uncertainties. In Proceedings of the 2018 IEEE International Conference on Systems, Man, and Cybernetics (SMC), Miyazaki, Japan, 7-10 October 2018; pp. 1386-1391.

19. Wu, Y.; Lau, V.K.N.; Tsang, D.H.K.; Qian, L.P.; Meng, L. Optimal Energy Scheduling for Residential Smart Grid with Centralized Renewable Energy Source. IEEE Syst. J. 2014, 8, 562-576. [CrossRef]

20. Carli, R.; Dotoli, M. A decentralized resource allocation approach for sharing renewable energy among interconnected smart homes. In Proceedings of the 2015 54th IEEE Conference on Decision and Control (CDC), Osaka, Japan, 15-18 December 2015; pp. 5903-5908.

21. Pilling, R.; Chang, S.; Luh, P.; Pilling, R.; Chang, S.C.; Luh, P.B. Shapley Value-Based Payment Calculation for Energy Exchange between Micro- and Utility Grids. Games 2017, 8, 45. [CrossRef]

22. OMIE. Available online: http://www.omel.es/pt/inicio (accessed on 30 April 2019). 